\title{
Effects of Photon Energy Spectrum on the Photocurrent of Hydrogenated Amorphous Silicon Thin Film Transistor by Using Frequency Filters
}

\author{
Eou Sik $\mathrm{Cho}^{\dagger}$ and Sang Jik Kwon \\ Department of Electronics Engineering, Gachon University, Seongnam 461-701, Korea
}

Received November 12, 2012; Revised November 15, 2012 Accepted November 19, 2012

\begin{abstract}
Frequency filters with various filtering wavelengths were used in the photoelectric characterization of hydrogenated amorphous silicon thin film transistor (a-Si:H TFT) and the experimental results were described and analyzed in terms of the photon energy spectral characteristics calculated from the integration of the photon energy and the spectral intensity of transmitted backlight through the filters at each wavelength. From the comparison of the photocurrents and the calculated photon energy spectrums for the filtered ranges of wavelength, it was possible to conclude that the photocurrents are closely related to the photon energy spectrums of the backlight.
\end{abstract}

Keywords: Frequency filter, Hydrogenated amorphous silicon thin film transistor (a-Si:H TFT), Photon energy spectral characteristics, Backlight, Photocurrent

\section{INTRODUCTION}

Thin film transistor(TFT) with a partially etched hydrogenated amorphous silicon(a-Si:H) channel has been mainly fabricated and used as a driving device in each pixel of active matrix display such as a thin film transistor liquid crystal display(TFT-LCD). Recently, the active photo mask process and source/drain photo mask process were merged into one photo mask process step for cost reduction in the fabrication of TFT panels, and it has been indispensable for a portion of the a-Si:H layer of TFT to be directly exposed to illumination from a backlight as a result of $\mathrm{O}_{2}$ ashing on source/drain electrodes and the subsequent wet etch process $[1,2]$. The a-Si:H TFT fabricated by the reduced photo mask process has characteristics of larger photocurrents than conventional a-Si:H TFT because more electron-hole pairs are generated in the exposed a-Si:H region by the photoelectric effect from backlight systems and their movements lead to a

${ }^{\dagger}$ Author to whom all correspondence should be addressed: E-mail: es.cho@gachon.ac.kr

Copyright @2013 KIEEME. All rights reserved.

This is an open-access article distributed under the terms of the Creative Commons Attribution Non-Commerciad License (http://creativecommons.org/licenses/by-nc/3.0) which permits unrestricted noncommercial use, photocurrent in the TFT. Moreover, the photocurrent of the TFT panel gets larger and larger as a result of the demand of the high luminous backlight systems for the application to a TV system. Therefore, it is necessary to perform an analysis on the mechanism of the photocurrent because the high photocurrent can produce effects on the optical characteristics of TFT-LCD, such as the degradation of contrast ratio, flickering, and image sticking. In the analysis of the generation of electron-hole pairs in a-Si:H layer to reduce the photocurrent, there has been a lot of research on the fabrication process condition of a-Si:H TFT [35]. However, it is not easy to change the process conditions of aSi:H TFT because of the unexpected side effects on the electrical characteristics of a-Si:H TFT.

Therefore, it is suggested that the spectral characteristics of the backlight system are used and modulated for the analysis of the photocurrent of a-Si:H TFT. In this article, the photocurrent of a-Si:H TFT was obtained for various spectral characteristics of backlight and the results were described in terms of the effect of the specific wavelength of backlight. The relationship between the spectral characteristics of the backlight and the photocurrent of a-Si:H TFT was analyzed by using the concept of a photon energy spectrum of backlight.

For the analysis of the effect of photon energy spectrum of backlight on photocurrent of a-Si:H TFT, a frequency filter was 


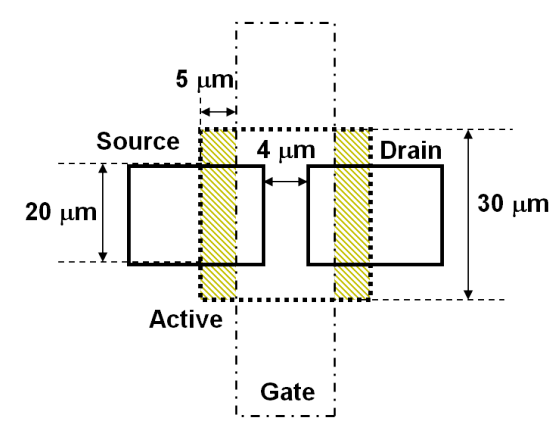

Fig. 1. A schematic diagram of the mask layout of TFT channel. The dashed layers are directly exposed to the backside illumination.

inserted in the photoelectric characterization of a-Si:H TFT. A cathode cold fluorescent lamp(CCFL) system was used as backlight and four different frequency filters were used for the filtering of the backlight. When a backside illuminated light goes through the frequency filter, the transmittance of the light is controlled in a restricted range of wavelength. It was possible to investigate the relationship between the specific range of wavelengths of transmitted light and the photoelectric effect of a-Si:H layer of TFT. From the comparison of the photon energy spectral and electrical results for various frequency filters, the cause and effect of the photocurrent of a-Si:H layer will be investigated and described.

\section{EXPERIMENTS}

A back channel etched inverted staggered a-Si:H TFT was fabricated by using a conventional 5 mask process $[6,7]$. To obtain a larger photocurrent as an effect of a reduced 4 photo mask process in the fabrication of TFT panel, a protruded a-Si:H region was designed to be exposed to the illumination from backlight as shown in the dashed area of Fig. 1. The width and the length of the channel were designed as $20 \mu \mathrm{m}$ and $4 \mu \mathrm{m}$, respectively.

Transfer characteristics were measured by using Agilent 4156C and a CCFL backlight. Four different frequency filters with green colors were used in the measurement and they were inserted between the TFT substrate and CCFL backlight during the measurement of photoelectric characteristics as shown in Fig. 2. MiNOLTA CD-100A and Photo Research 670 Spectrascan Colorimeter were used in the measurement of the color characteristics and the spectral characteristics of the backlight, respectively.

\section{RESULTS AND DISCUSSION}

Table 1 shows the color characteristics of the transmitted lights through the various frequency filters used in the experiment. The CCFL backlight showed the luminance value of $1,698 \mathrm{~cd} / \mathrm{m}^{2}$. For the green 1 , green 2 , green 3 , and green 4 filters, the luminance value of the transmitted light was 1079, 804.1, 756.4, and 655.73 $\mathrm{cd} / \mathrm{m}^{2}$, respectively. From the CIE chromaticities of the transmitted lights, it is possible to investigate the slight difference in the transmittance of the backlight through each frequency filter on a specific range of wavelength. The overall transmittance of the filters was $62.16,44.99,43.26$, and $36.95 \%$ for green 1 , green 2 , green 3 , and green 4 filters, respectively. The ratios of the luminance of the transmitted light through each filter to that of the CCFL backlight without filters were calculated as 63.5, 47.3, 44.5, and $38.5 \%$ for green 1 , green 2 , green 3 , and green 4 filters, respectively. The ratios were almost the same as the transmittances

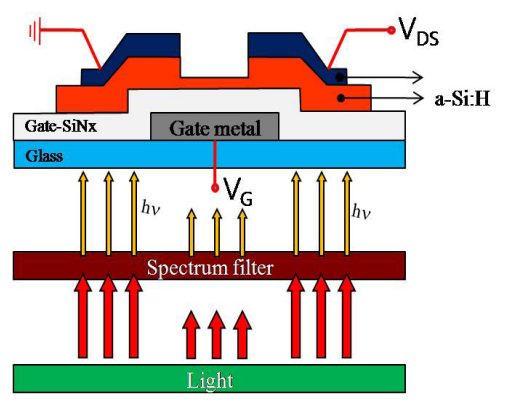

Fig. 2. A schematic diagram of the mask layout of TFT channel. The dashed layers are directly exposed to the backside illumination.

Table 1. Color characteristics of the transmitted light through the various frequency filters.

\begin{tabular}{cccc}
\hline Filter & $\begin{array}{c}\text { Luminance } \\
\left(\mathrm{cd} / \mathrm{m}^{2}\right)\end{array}$ & $\begin{array}{c}\text { Transmittance } \\
(\%)\end{array}$ & $\begin{array}{c}\text { CIE chromaticity } \\
(\mathrm{x}, \mathrm{y}, \mathrm{z})\end{array}$ \\
\hline None & 1,698 & 100 & $(0.26,0.28,0.46)$ \\
\hline Green 1 & 1,079 & 62.16 & $(0.26,0.43,0.31)$ \\
\hline Green 2 & 804.1 & 44.99 & $(0.25,0.59,0.16)$ \\
\hline Green 3 & 756.4 & 43.26 & $(0.19,0.48,0.33)$ \\
\hline Green 4 & 655.73 & 36.95 & $(0.20,0.56,0.24)$ \\
\hline
\end{tabular}

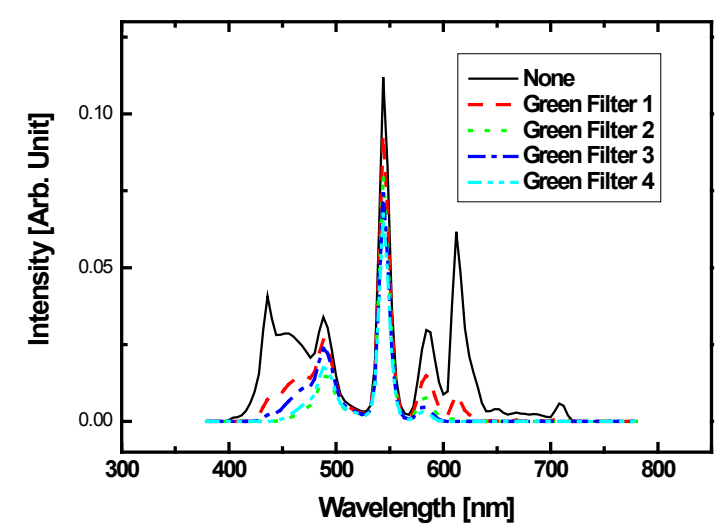

Fig. 3. Spectral characteristics of the transmitted lights through the frequency filters used in the experiment.

of the various frequency filters.

Figure 3 shows the spectral characteristics of a CCFL backlight when the lights are transmitted through the frequency filters used in the experiment. Without any inserted frequency filter, the three apparent intensity peaks were investigated at $436 \mathrm{~nm}$ (blue), $544 \mathrm{~nm}$ (green), and $612 \mathrm{~nm}$ (red) and the two small intensity peaks were additionally investigated at about $490 \mathrm{~nm}$ and $580 \mathrm{~nm}$. For the transmitted lights through the green filters, the spectral characteristics have apparent intensity peaks at $544 \mathrm{~nm}$ and relatively apparent peaks at about 488 $\mathrm{nm}$ and $584 \mathrm{~nm}$. In the case of green filter 1, a small peak was also investigated at $612 \mathrm{~nm}$. The magnitudes of intensity peaks of the transmitted light through the filters were much smaller than those of the non-filtered CCFL backlight because of the lower transmittance of the filters. In the case of green filter 1 , the magnitudes of intensity peaks of the transmitted light were higher than those through the other filters. The intensity peaks were proportional to the transmittance of the green filters. However, in the case of green filter 3, the magnitude of intensity peak at $488 \mathrm{~nm}$ was higher than those of green filter 2 . The 


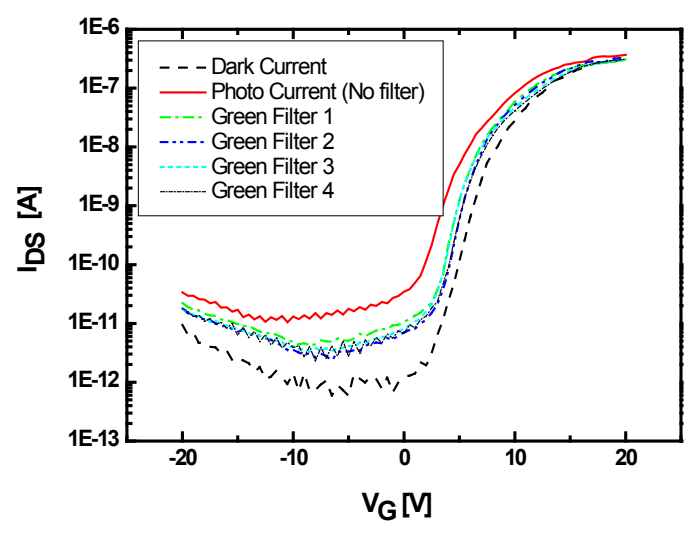

Fig. 4. Transfer characteristics of a-Si:H TFT under the transmitted lights though the frequency filters of Fig. 2.

spectral characteristics also show the intensity of the transmitted light through green filter 3 has a larger magnitude than that through green filter 2 in a range of the wavelength from $424 \mathrm{~nm}$ to $528 \mathrm{~nm}$.

Figure 4 shows the transfer characteristics of the fabricated TFT under the backside illumination through the various frequency filters. Without any illumination from the CCFL backlight, an on/off current ratio of $4.14 \times 10^{5}$ was obtained when the on current and off current was defined as the current at the gate voltage of $20 \mathrm{~V}$ and $-8 \mathrm{~V}$, respectively. At the gate voltage below $-10 \mathrm{~V}$, the off-current was increased as a result of the effect of the hole current. The mobility and the threshold voltage was less than $0.2 \mathrm{~cm}^{2} / \mathrm{V}$.s and about $5 \mathrm{~V}$, respectively. With the backside illumination from the CCFL backlight, the photocurrent of the TFT was higher than the dark current without any illumination in the negative gate bias by about a factor of $10^{1}$ or more. The photocurrent was also higher than the dark current in the forward gate bias as a result of the protruded active layer outside gate electrode as shown in Fig. 1. For the transmitted lights through the various frequency filters, the photocurrents were smaller than those of the non-filtered backlight because of the luminous transmittance of filters and were a little larger than the dark current. However, there were a little difference in the photocurrents of the transmitted light through green filter 3 was higher than that through green filter 2, in spite of the different luminance values of transmitted lights as shown in Fig. 5. From the results, it is possible to explain that the photocurrent is related to both the intensity of the incident light at a lower wavelength of light and to the absorption coefficient of a-Si:H layer $[6,8]$

For more elaborate descriptions on the relationship between the photocurrents and the spectral characteristics of the lights, the photon energy of the incident light was considered. When the photon energy of the incident light is larger than the energy bandgap of the layer, it is possible for the light to be absorbed. Without considering the absorption coefficient of a-Si:H layer as a variable to the wavelength, it is possible to postulate that the incident lights are absorbed in the layer in a range of the visible rays below about $700 \mathrm{~nm}$ because the energy bandgap of a-Si:H layer is about 1.5 1.8 eV. From the above postulations, photon energies absorbed in the a-Si:H TFT were calculated for the transmitted lights of Fig. 3 by using the multiplication of each photon energy, absorption coefficient, and spectral intensity at a wavelength and also the integration for a region of optical wavelength of the backlight as below. Because the spectral intensities are weak and insignificant in a region of above $700 \mathrm{~nm}$ from Fig. 3 , it is possible to integrate from $380 \mathrm{~nm}$ to $780 \mathrm{~nm}$ without an ad-

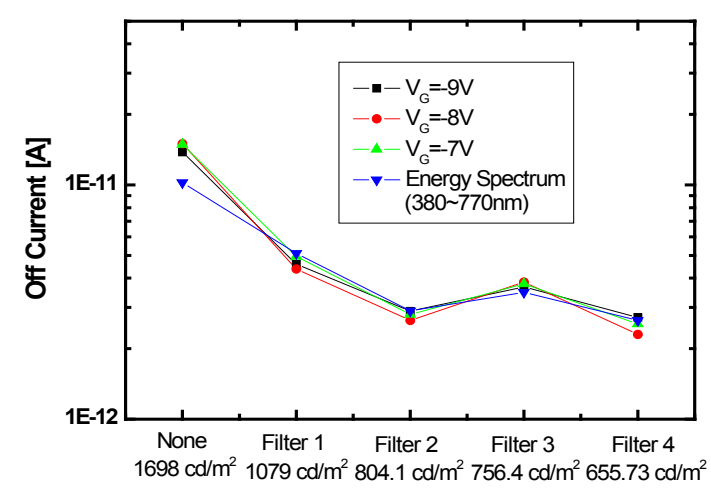

Fig. 5. Comparison of the fitted photon energies of the transmitted lights through the frequency filters and the off-current at the gate voltage of $-9 \mathrm{~V},-8 \mathrm{~V}$, and $-7 \mathrm{~V}$ from Fig. 3.

ditional upper or lower limit.

$$
\begin{aligned}
& \text { Absorbed photon energy }= \\
& \int_{380 \mathrm{~nm}}^{780 \mathrm{~nm}} \frac{h c}{\lambda} \text { (absorbed coefficient) (spectral intensity) } \mathrm{d} \lambda
\end{aligned}
$$

As shown in Fig. 3, the spectral characteristics were disintegrated into a lot of segments at a spacing of $4 \mathrm{~nm}$. The absorption coefficient was considered as a unity, or constant. From the equation (1), the areas of photon energy spectrums were calculated as $5.12 \times 10^{-28}, 2.55 \times 10^{-28}, 1.45 \times 10^{-28}, 1.74 \times 10^{-28}$, and $1.32 \times 10^{-30}$ for the non-filtered backlight, and the green filters $1,2,3$, and 4 , respectively.

Figure 5 shows that the comparison of the integrated photon energies the off-current defined at the gate voltage of $-9 \mathrm{~V},-8 \mathrm{~V}$, $-7 \mathrm{~V}$ obtained from Fig. 4 for the more detailed analysis. For the fitting of the photon energies to the off-currents, a constant weighting factor was multiplied to the photon energies. Based on the outcomes, it is possible to conclude that the integrated photon energies are comparatively well fitted to the experimental photoelectric off-current in spite of the simplification of the absorption coefficient. For the minor difference in the non-filtered backlight between the off currents and the calculated photon energy, it is speculated that the simplification of the absorption coefficient diminishes the calculated photon energy at a lower wavelength in the integration of the photon energy.

\section{CONCLUSIONS}

Various frequency filters were applied to the photoelectric characterization of a-Si:H TFT and the obtained photocurrents through the filters were not directly related to the luminance of the transmitted backlight. From the photocurrents of a-Si:H TFT and the photon energy spectrums calculated from the spectral characteristics of the transmitted backlight through the filters, it was possible to conclude that the photocurrent is closely related to the integrated photon energy spectral characteristics in a range of visible rays. For the improvement of the image quality of TFT-LCD, the spectral characteristics of the backlight should be considered as an important factor to reduce the photocurrent of a-Si:H TFT. 


\section{ACKNOWLEDGMENTS}

This work was supported by the Korean Research Foundation (KRF) grant funded by the Korean government(MEST) (No. 20120003655). This work was also supported by Light Measurement Solution (LMS) in the measurement of spectral characteristics of light.

\section{REFERENCES}

[1] S. Y. Yoo, H. S. Choi, J. I. Ryu, W. B. Lee, and J. Y. Lee: Proc. IDW'05, p.1121, Takamatsu, Japan, Dec 6-9 (2005).

[2] S. Kido, M. Yamashita, K. Akagami, and Y. Mutayama: Proc. IDW'05, p.2005, Takamatsu, Japan, Dec 6-9 (2005).
[3] C.-Y. Liang, F.-Y. Gan, P.-T. Liu, F. S. Yeh, S. H.-L. Chen, and T.-C Chang, IEEE Electron Device Lett. 27, 978 (2006) [DOI: http:// dx.doi.org/10.1109/LED.2006.886418].

[4] Y. J. Choi, B. C. Lim, I. K. Woo, J. I. Ryu, and J. Jan, J. Non-Cryst Solids. 266, 1299 (2000) [DOI: http://dx.doi.org/10.1016/S00223093(99)00941-2].

[5] J. H. Choi, C. S. Kim, B. C. Lim, and J. Jang, IEEE Trans. Electron Dev. 45, 2074 (1998) [DOI: http://dx.doi. org/10.1109/16.711377].

[6] S. J. Hong, S. J. Kwon, and E. S. Cho, Jap. J. Appl. Phys. 47, 6974 (2008) [DOI: http://dx.doi.org/10.1143/JJAP.47.6974].

[7] M. J. Powell, IEEE Trans. Electron Dev. 36, 2753 (1989) [DOI: http://dx.doi.org/10.1109/16.40933].

[8] E. S. Yang: Microelectronics Devices (McGraw-Hill, New York, 1994). 